\title{
A Structural Approach to Adaptive Inverse Halftoning for Document Images
}

\author{
Hirobumi Nishida \\ Ricoh Image Appliance Lab, 1-1-17 Koishikawa, Bunkyo-ku, Tokyo 112-0002, Japan \\ hn@src.ricoh.co.jp
}

\begin{abstract}
This paper describes an efficient algorithm for inverse halftoning of scanned color document images to resolve problems with interference patterns such as moiré and graininess when the images are displayed or printed out. The algorithm is suitable for software implementation and useful for high quality printing or display of scanned document images delivered via networks from unknown scanners. A multi-resolution approach is used to achieve practical processing speed under software implementation. Through data-driven, adaptive, multi-scale processing, the algorithm can cope with a variety of input devices and requires no information on the halftoning method or properties (such as coefficients in dither matrices, filter coefficients of error diffusion kernels, screen angles, or dot frequencies). Effectiveness of the new algorithm is demonstrated through real examples of scanned color document images.
\end{abstract}

\section{Introduction}

When we display or print out scanned document images (obtained through image input devices) without applying any image processing operations to them or after scaling or rotating them, we often observe image distortions or degradations such as moiré phenomena and graininess. Halftone areas on printed document images consist of dot patterns generated by screening methods or error-diffusion algorithms. Such dot patterns often interact with image processing operations (halftoning, scaling, etc.) performed by output devices such as printers or displays. This problem of image degradation can be resolved if halftone areas composed of dot patterns are transformed to ideal continuous-tone representations. Such an operation of restoring ideal continuous-tone representations from halftone dot patterns is usually referred to as inverse halftoning. In designing inverse halftoning algorithms for scanned document images, we require that characters, graphics such as line drawings, and significant edges be preserved without being blurred.

Such processing for improving image quality has been embedded into imaging devices such as copiers. In particular, there have been a number of methods for inverse halftoning. Methods applicable to halftone patterns generated by screening methods and error-diffusion algorithms are nonlinear permutation filters [1], a combination of Gaussian filters, median filters, and edge enhancement [2], and a wavelet-based method [3]. Statistical approaches include a convex-projection method using knowledge on error-diffusion kernels and nonlinear optimization [4], MAP projection and estimation of error-diffusion kernels using a modified LMS method [5], Bayesian 


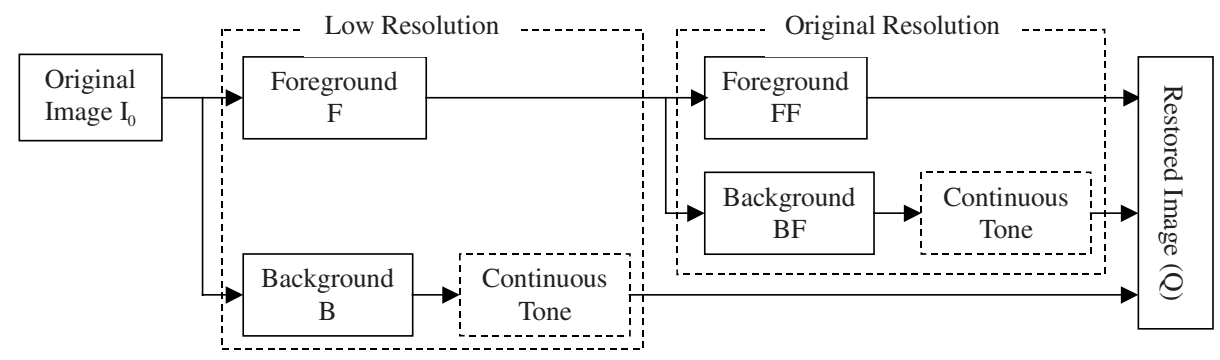

Fig. 1. Overview of the proposed algorithm.

estimation with nonlinear optimization [6], and MAP projection using wavelet [7]. However, they require expensive computation because iterative computations are involved. Kite et al. [8], Roetling [9,10], Miceli and Parker [11], and Chen and Hang [12] employ space-variant linear filters along with multi-scale gradient information. A hybrid LMS-MMSE method [13] and an LUT-based method [14] are computationally inexpensive.

In network and Internet environments, digital image data acquired through any input device can be transmitted to remote sites over networks, and the receiver can display or print out the image data delivered from unknown devices. Under these conditions, image data must be processed with software on individual PCs so that images can be printed or displayed in adequate quality without annoying distortions or degradations. Now, new technical requirements have emerged as follows:

- Practical processing speed must be achieved under software implementation.

Under network environments, image data delivered via networks from remotely located input devices are often processed on individual PCs for improving image quality when the data are displayed or printed out. Practical processing speed must be achieved under software implementation on PCs. If space-variant linear filters or wavelet-based inverse halftoning algorithms are implemented with software, computation time is not acceptable when they are applied to scanned color document images of A4 or letter size.

- Image processing systems must easily adapt to a variety of input devices.

In image processing systems embedded into stand-alone imaging devices where input and output media are integrated, algorithms and parameters are designed so that they fit the color characteristics, resolution, and frequency characteristics (MTF) of particular devices. However, image-processing systems optimal for a particular imaging device are not necessarily effective for other devices with different characteristics. Under network environments, there are large variations in characteristics of imaging devices, and the characteristics are even unknown when image data are transmitted from a remote site. Therefore, image-processing systems and algorithms must easily adapt to a variety of input devices. For instance, satisfactory image quality should be achieved for image data obtained through an unknown scanner just by setting a few, simple parameters.

- Information on the halftoning methods or properties is not required.

Specific methods for inverse halftoning can be employed if information is available on the halftoning method or properties (such as coefficients in dither matrices, 


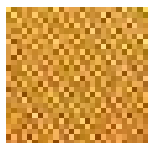

(a)

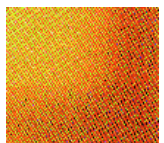

(b)

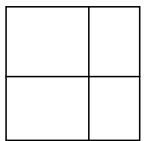

(c)

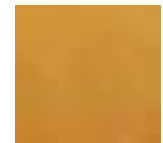

(d)

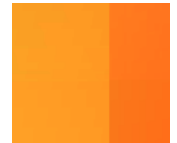

(e)

Fig. 2. (a) The background color is uniform or smoothly changes over the region. (b) The background consists of two or more distinct colors. (c) Location of windows. (d) Continuous tone representation for (a). (e) Continuous tone representation for (b).

filter coefficients of error diffusion kernels, screen angles, and dot frequencies). However, our targets are scanned images of books, magazines, journals, and newspapers that have been printed through unknown printing processes. Furthermore, characteristics of halftone dot patterns cannot be extracted when the input device has a poor response to high frequencies or the image resolution is inadequate. Therefore, blind methods are required without depending on the type or properties of halftoning processes.

In this paper, we describe an efficient algorithm for inverse halftoning of scanned color document images to resolve problems of interference patterns such as moiré and graininess when the images are displayed or printed out. The algorithm is suitable for software implementation and useful for high quality printing or display of scanned document images delivered via networks from unknown scanners. A multi-resolution approach is used to achieve practical processing speed under software implementation. Through data-driven, adaptive, multi-scale processing, the algorithm can cope with a variety of input devices and requires no information on the halftoning method or properties (such as coefficients in dither matrices, filter coefficients of error diffusion kernels, screen angles, or dot frequencies). Effectiveness of the new algorithm is demonstrated through real examples of scanned color document images.

This paper is organized as follows: An overview of the proposed algorithm is given in Sect. 2. The details of the algorithm are presented in Sect. 3. Examples and experimental results are shown in Sect. 4. Sect. 5 is the conclusion.

\section{Outline}

We outline the approach to resolving the three problems mentioned in Sect. 1. The overall architecture is shown in Fig. 1. A multi-resolution approach is used to achieve practical processing speed under software implementation. In each resolution, through data-driven, adaptive, multi-scale processing, the algorithm can cope with a variety of input devices and requires no information on the halftoning method or properties.

\section{Multi-resolution Analysis}

According to the Mixed Raster Content (MRC) Imaging Model, a color document image is composed of the following two components:

- Foreground: text, graphics (line drawings, in particular), and significant edges

- Background: other areas (continuous tone areas)

There are few significant edges in the background, which can be compressed to low resolution such as 100 dpi without perceptual degradation by human eyes, while 


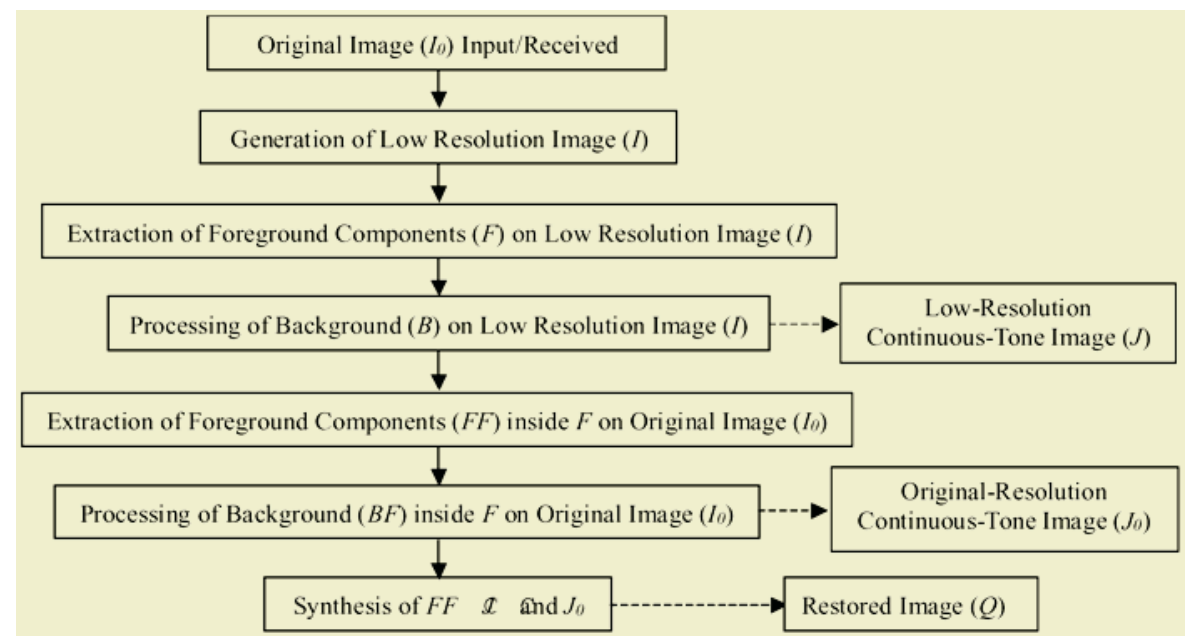

Fig. 3. Flowchart for the proposed algorithm.

high resolution is required to preserve high frequency components for the foreground. In terms of image representation, quite different methods and viewpoints are required for these two components. Therefore, the background can be processed on a reduced image in low resolution, and consequently, the overall throughput can be improved significantly.

The multi-resolution analysis proceeds as follows: First, a reduced image $I$ in low resolution is generated from the original image $I_{0}$. On the reduced image $I$, foreground components $F$ are extracted based on local features. The remaining areas are set to the background $B$. The background $B$ is transformed to a continuous-tone representation.

Next, the foreground components $F$ are processed in the original resolution. Inside the area $F$, foreground components $F F$ are further extracted based on local features, and the remaining areas are set to background $B F$. The background $B F$ is also transformed to a continuous-tone representation.

Finally, the background components $B$ represented as continuous tones, the foreground components $F F$, and the background components $B F$ represented as continuous tones are synthesized together to obtain the restored image.

\section{Processing of Background: Multi-scale Analysis from Coarse to Fine}

The background components $(B$ and $B F$ ) are transformed to continuous-tone representations. If the halftoning method and parameters for generating dot patterns are known, filter banks with appropriate support size can be prepared and space-variant linear filters can be applied to each pixel according to local image features. However, there is no information available to decide appropriate support size when characteristics of the input device are unknown. Therefore, multi-scale analysis from coarse to fine is employed.

In the background areas that remain after extracting the foreground, we consider a region $R$ of size $W \times W$. There should be no significant edges, characters, or graphics in this region $R$. Therefore, if size $W$ and location of $R$ are appropriate, colors of pixels in region $R$ can be replaced with the average color in $R$. For instance, for region $\mathrm{R}$ 
shown in Fig. 2(a), an appropriate continuous tone representation can be obtained by this operation as shown in Fig. 2(d).

However, distortions could be introduced when $W$ is too large or location of $R$ is inappropriate. For region $R$ shown in Fig. 2(b), if windows are located as shown in Fig. 2(c), this operation leads to the representation shown in Fig. 2(e), where distortions are observed. To resolve this problem, we need to detect distortions, redoing the transformation with smaller $W$.

The edges on the transformed continuous-tone image should be a subset of edges on the original image, because smoothing or blurring has been applied. As far as the transformation process proceeds relevantly, there should not be edges on the transformed image that do not exist on the original. For each pixel, the edge magnitude on the transformed image should not exceed that on the original. As shown in Fig. 2(e), if there are some edges in the transformed image that do not exist on the original, it implies that $W$ is too large or location of $R$ is inappropriate. To correct the distortions, the continuous-tone transformation is redone around the pseudo-edges with smaller $W$. Since it is difficult to set the appropriate scale $W$ adaptively for each pixel without a priori knowledge, a coarse-to-fine strategy is employed along with detection of anomalies on the transformed image based on the edge feature.

\section{Algorithm}

Following the overview presented in Sect. 2, we describe the algorithm in this section. A flowchart for the proposed algorithm is presented in Fig. 3. An example of the original image $I_{0}$ (400dpi) is shown in Fig. 4.

\section{Foregroung Extraction}

A reduced image $I$ in low resolution (100 to 200dpi) is generated from the original image $I_{0}$ by a simple local averaging method. The reduced image $I$ is smoothed with a linear filter for noise reduction. The selection of this filter depends on the frequency characteristics of the input image, but we have only to prepare just a few types of filters to deal with a wide range of characteristics. Furthermore, all pixels are initialized to background: $[i, j] \in B$ for all $i, j$. Foreground components $F$, as shown in Fig. 5 , are extracted from the smoothed reduced image $I$ by a locally adaptive thresholding technique, along with morphological dilation operations.

\section{Transformation of Background to Continuous Tone Representation}

The background areas $B$, as shown in white areas in Fig. 5, are transformed to a continuous tone representation through local, adaptive, multi-scale processing. Before the transformation, edge magnitude $E_{0}$ is calculated on the reduced image $I$.

\section{Generation of Initial Transformed Images}

The initial transformed image is generated for background components $B$. As shown in Fig. 6, for each horizontal scan line, runs of the maximum length wsz (typically $0.5 \mathrm{~mm}$ ) are constructed sequentially from left to right so that each run contains no foreground pixels inside. The average color is calculated for each run, and is set to 


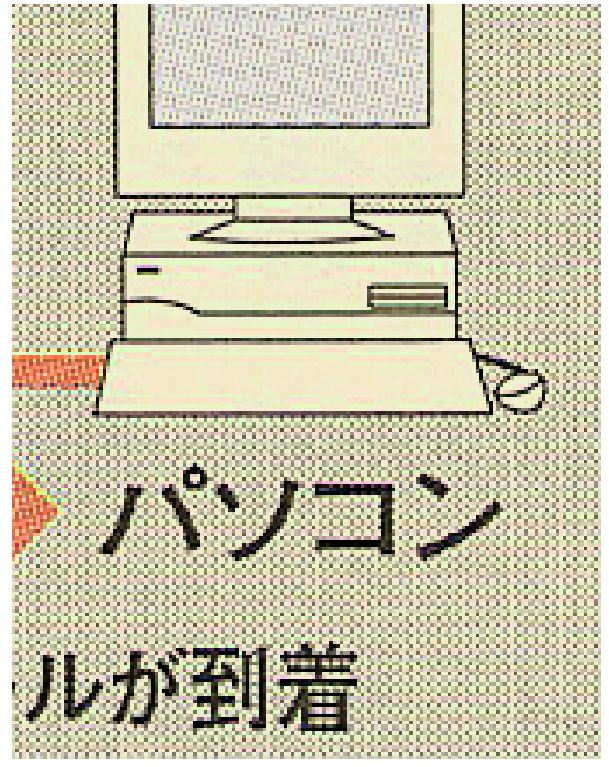

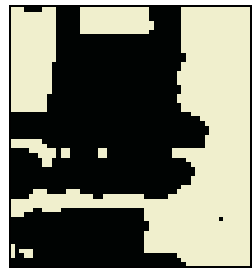

Fig. 5. Foreground components $F$ extracted from the reduced image $I$.

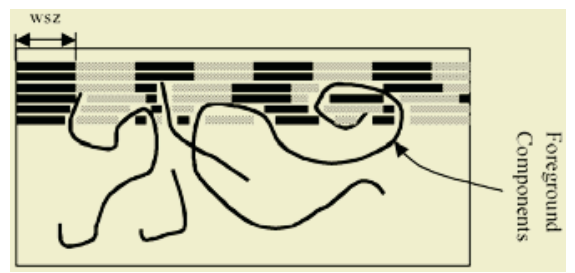

Fig. 6. Transformation into continuous tone representation.

Fig. 4. Original image $I_{0}$.

each pixel on the run. In this way, the continuous tone representation $C^{\prime}$ is obtained based on horizontal runs. Furthermore, the continuous tone transformation is applied to vertical runs of the generated image $C^{\prime}$ in the same way as horizontal runs. The initial transformed image $J$ is generated as a result of the continuous tone transformation for horizontal and vertical runs.

\section{Correction to Transformed Images}

The transformed image $J$ is corrected through adaptive, multi-scale analysis of edges on the transformed images, based on the principle described in Sect. 2.2.

(1) Reduce $w s z$ to the half. If $w s z$ is less than a predetermined size, then go to (7).

(2) Compute the edge magnitude image $E$ ' for the transformed image $J$.

(3) Compute the pseudo edge image $E_{1}$ from the difference between $E$ ' and $E_{0}$. For pixel $(i, j)$, if $E^{\prime}(i, j)>E_{0}(i, j)$, set $E_{1}(i, j)$ to "ON," otherwise "OFF."

(4) Using the new value of $w s z$, the continuous tone transformation is redone locally around "ON" pixels of $E_{1}$. A new transformed image $C$ is generated.

(5) On the transformed image $J$, colors of $(2 \cdot w s z-1) \times(2 \cdot w s z-1)$ pixels around each "ON" pixel on the pseudo edge image $E_{1}$ are replaced with those on the new transformed image $C$.

(6) Go to (1)

(7) End.

The correction is applied from a coarse scale to a fine scale by detecting distortions through the analysis of edge magnitude between the original and the transformed images. Fig. 7 shows the result of the series of processing embedded into the original image $I_{0}$. 


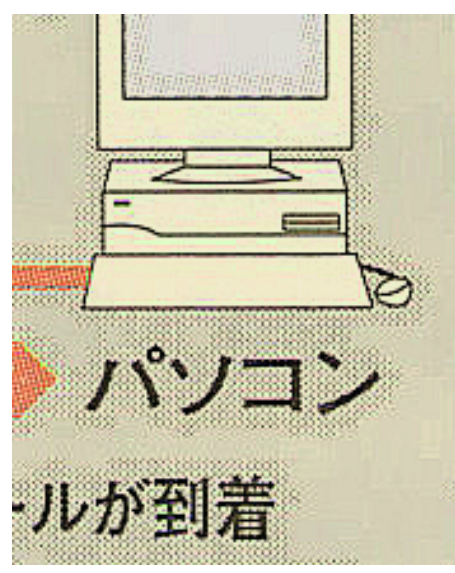

Fig. 7. Transformed image $J$ embedded into the original image.

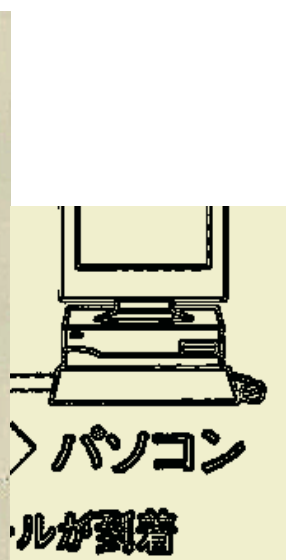

Fig. 8. Foreground components $F F$.

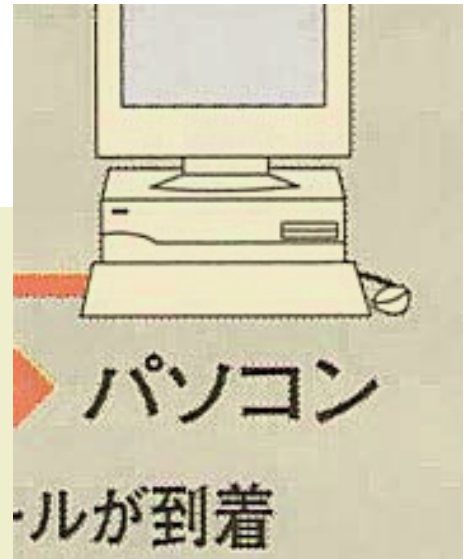

Fig. 9. Final result $Q$.

\section{Processing in the Original Resolution and Synthesis}

The foreground components $F$ extracted from the reduced image $I$ are processed in the original resolution in order to obtain a continuous tone transformation in fine detail. Note that, in Fig. 7, there are still some pixels that need to be transformed to a continuous tone representation, particularly around characters and line drawings.

Inside this area $F$, foreground components are extracted from the original image $I_{0}$ in the same way as described in Sect. 3.2. Let $F F$ be the area extracted as foreground (Fig. 8), and $B F$ be the remaining area. Furthermore, for the original image $I_{0}$, the area $B F$ is transformed to a continuous tone representation in the same way as described in Sect. 3.3, and the transformed image $J_{0}$ is obtained.

The continuous tone representation $J$ of the background components $B$, the foreground components $F F$, and the continuous tone representation $J_{0}$ of the background components $B F$ are synthesized together to obtain to the final restored image $Q$. Fig. 9 shows the final result for the original image shown in Fig. 4.

\section{Experimental Results}

The proposed algorithm has been evaluated with real scanned document images to verify the effectiveness in terms of processing speed and image quality.

Computation time in a low-end environment (Pentium III, 933MHz $\square 256 \mathrm{MB}$ ) has been measured for 24bit full-color images of scanned documents of A4-size $(210 \mathrm{~mm}$ by $297 \mathrm{~mm})$, in comparison with space-variant linear filters with support size $5 \times 5$ and 7x7. Table 1 shows the quite promising results where remarkable acceleration can be observed for the proposed method. The result implies that practical processing speed can be achieved under software implementation.

Some real examples are presented for scanned color document images. Fig. 10(a) is the original image generated by the Floyd-Steinberg error-diffusion algorithm and 

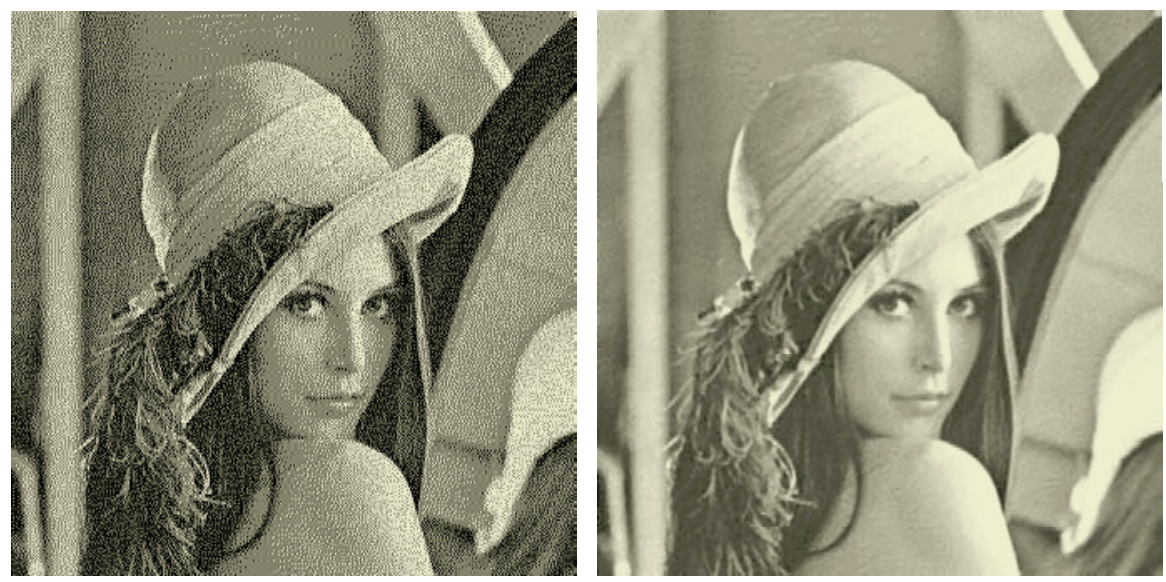

Fig. 10. Original image and result of the proposed algorithm.

Fig. 10(b) is the result of the continuous tone transformation. Because of the datadriven, adaptive, multi-scale processing, there is no perceivable distortion in the transformed image. Fig. 11(a) is a text image on top of grainy background and Fig. 11(b) is the result of the continuous tone transformation. The graininess of the background is completely resolved without blurring character strokes. Fig. 12(a) contains fine line drawings that are likely to be blurred by inverse halftoning and Fig. 12(b) is the result of the continuous tone transformation. Fine details are still preserved in the transformed image.

\section{Conclusion}

We have described an efficient algorithm for inverse halftoning of scanned color document images to resolve problems of interference patterns such as moiré and graininess when the images are displayed or printed out. The algorithm is suitable for software implementation and useful for high quality printing of scanned document images delivered via networks from unknown scanners. A multi-resolution approach is used to achieve practical processing speed under software implementation. Through data-driven, adaptive, multi-scale processing, the algorithm can cope with a variety of input devices and requires no information on the halftoning method or properties. Effectiveness of the new algorithm has been demonstrated through real examples of scanned color document images.

Table 1. Computation time in a low-end environment for full-color images of scanned documents of A4-size, in comparison with space-variant linear filters.

\begin{tabular}{|c|c|c|c|}
\hline \multirow{2}{*}{ Resolution dpi } & \multirow{2}{*}{$\begin{array}{c}\text { Proposed } \\
\text { Method }\end{array}$} & \multicolumn{2}{|c|}{ Space-Variant Linear Filter } \\
\cline { 3 - 4 } & $3 \mathrm{sec}$. & $5 \mathrm{x} 5$ & $7 \mathrm{x} 7$ \\
\hline 200 & $7 \mathrm{sec}$. & $46 \mathrm{sec}$. & $16 \mathrm{sec}$. \\
\hline 400 & & $67 \mathrm{sec}$. \\
\hline
\end{tabular}




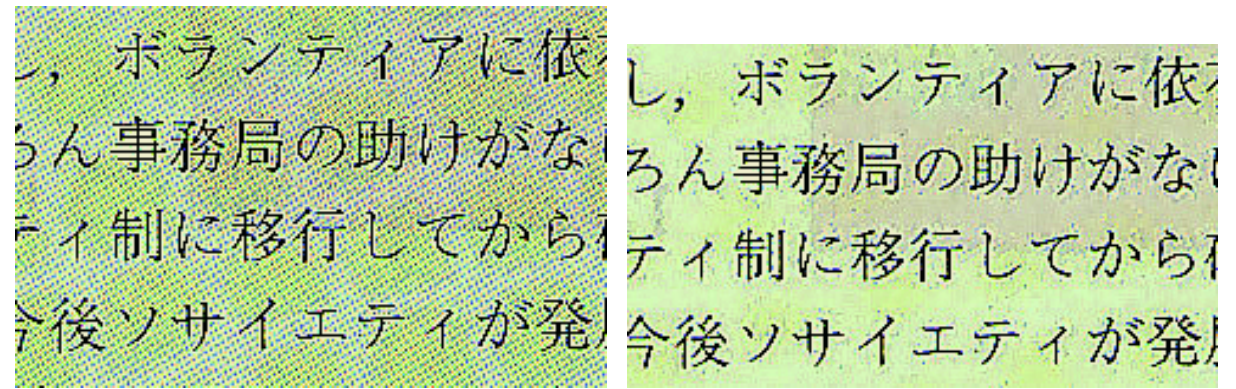

Fig. 11. Original image and result of the proposed algorithm.
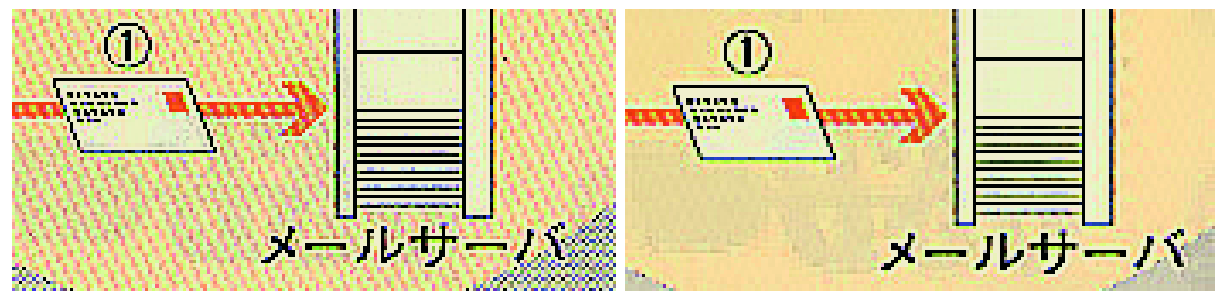

Fig. 12. Original image and result of the proposed algorithm.

\section{References}

1. Y. Kim, G. Arce, and N. Grabowski, Inverse halftoning using binary permutation filters, IEEE Trans. Image Processing, 4, 1296 - 1311, 1995.

2. N. Damera-Venkata, T.D. Kite, M. Venkataraman, and B.L. Evans, Fast blind inverse halftoning, Proc. 1998 IEEE International Conference on Image Processing, 64 - 68, 1998.

3. J. Luo, R. de Querioz, and Z. Fan, A robust technique for image descreening based on the wavelet transform, IEEE Trans. Signal Processing, 46, 1179 - 1194, 1998.

4. S. Hein and A. Zakhor, Halftone to continuous-tone conversion of error-diffusion coded images, IEEE Trans. Image Processing, 4, 208 - 216, 1995.

5. P. Wong, Inverse halftoning and kernel estimation for error diffusion, IEEE Trans. Image Processing, 4, 486 - 498, 1995.

6. R. Stevenson, Inverse halftoning via MAP estimation, IEEE Trans. IP, 6, 574 - 583, 1997.

7. Z. Xiong, M. Orchard, and K. Ramchandran, Inverse halftoning using wavelet, IEEE Trans. Image Processing, 8, 1479 - 1483, 1999.

8. T.D. Kite, N. Damera-Venkata, B.L. Evans, and A.C. Bovik, A fast, high-quality inverse halftoning algorithm for error diffused halftones, IEEE Trans. IP, 9, 1583 - 1592, 2000.

9. P. Roetling, US Patent 5343309, August 1994.

10. P.G. Roetling, US Patent 4630125, 1986.

11. C. Miceli and K.J. Parker, Inverse halftoning, Journal of Electronic Imaging, vol. 1, pp. 143 - 151, April 1992.

12. L.-M. Chen and H.-M. Hang, An adaptive inverse halftoning algorithm, IEEE Trans. Image Processing, 6, 1202 - 1209, 1997

13. P.-C. Chang, C.-S. Yu, and T.-H. Lee, Hybrid LMS-MMSE inverse halftoning technique, IEEE Trans. Image Processing, 10, 95 - 103, 2001.

14. M. Mese and P.P. Vaidyanathan, Look-up table (LUT) method for inverse halftoning, IEEE Trans. Image Processing, 10, 1566 - 1578, 2001. 